\title{
Clinical Trial of Chronic Implantation of Suprachoroidal-Transretinal Stimulation System for Retinal Prosthesis
}

\author{
Takashi Fujikado*, Motohiro Kamei ${ }^{1}$, Hirokazu Sakaguchi' ${ }^{1}$, \\ Hiroyuki Kanda, Takeshi Morimoto, Yasushi Ikuno ${ }^{1}$, Kentaro Nishida ${ }^{1}$, \\ Haruhiko Kishima², Tomoyuki Maruo², Hajime Sawai ${ }^{3}$, \\ Tomomitsu Miyoshi ${ }^{3}$, Koji Osawa ${ }^{4}$ and Motoki Ozawa ${ }^{4}$ \\ Department of Applied Visual Science, Osaka University Graduate School of Medicine, \\ 2-2 Yamadaoka, Suita, Osaka 565-0871, Japan \\ 'Department of Ophthalmology, Osaka University Graduate School of Medicine, \\ 2-2 Yamadaoka, Suita, Osaka 565-0871, Japan \\ ${ }^{2}$ Department of Neurosurgery, Osaka University Graduate School of Medicine, \\ 2-2 Yamadaoka, Suita, Osaka 565-0871, Japan \\ ${ }^{3}$ Department of Integrative Physiology, Osaka University Graduate School of Medicine, \\ 2-2 Yamadaoka, Suita, Osaka 565-0871, Japan \\ ${ }^{4}$ Nidek Co., 34-14 Maehama, Hiroishi, Gamagori, Aichi 443-0038, Japan
}

(Received December 9, 2011; acceped January 30, 2012)

Key words: retinal prosthesis, retinitis pigmentosa, artificial vision, clinical trial, suprachoroidaltransretinal stimulation

Retinal prosthesis is an implantable medical device to reconstruct the sense of sight for blind individuals. We have been developing a new type of retinal prosthesis called suprachoroidal-transretinal stimulation (STS), whereby an electrode array is not attached to the retina directly. To evaluate the feasibility and safety of the retinal prosthesis using STS, a clinical trial was performed. We have developed an internal device for chronic implantation. It consists of a 49-channel electrode array that has 9 active electrodes. The retinal prosthesis was implanted in two patients with advanced retinitis pigmentosa (RP). Follow-up periods after implantation were five ( $\mathrm{Pt} 1)$ and seven $(\mathrm{Pt} 2)$ weeks. No significant adverse event was observed in either patient after the surgical procedures. A functional test revealed that the detection or discrimination of objects was possible using the device, and a pulse frequency of around $20 \mathrm{~Hz}$ is the most effective in evoking phosphene. These clinical examinations showed that the retinal prosthesis with the STS system is safe and feasible for artificial vision.

"Corresponding author: e-mail: fujikado@ophthal.med.osaka-u.ac.jp 


\section{Introduction}

Retinitis pigmentosa (RP) is one of the leading causes of blindness. ${ }^{(1)}$ There is no treatment for this disease. To restore some vision, stimulating the residual functional retinal neurons by electrical currents delivered through an electrode array is being studied. This is called "retinal prosthesis".

There are three ways of stimulating the retina for a retinal prosthesis: subretinal stimulation,(2) epiretinal stimulation, ${ }^{(3)}$ and suprachoroidal-transretinal stimulation (STS). ${ }^{(4-6)}$ In subretinal stimulation, the electrode array is placed in the subretinal space. In epiretinal stimulation, the electrode array is attached to the vitreous side of the retina. In STS, the electrode array is placed in a scleral pocket or choroidal membrane and the return electrode in the vitreous cavity. STS was originally designed by our group. The main feature of STS is that the electrode array is not directly attached to the retina. Therefore, it can minimize damage to the retina during the electrode implantation surgery.

For clinical application of the retinal prosthesis with STS, it is important to evaluate its feasibility and safety through clinical trial. For that reason, we have developed a microelectrode-STS system that can be chronically implanted into the patient. The purpose of this study is to evaluate the safety and stability of the system when it is implanted in a patient with RP.

\section{Materials and Methods}

\subsection{Subjects}

Two patients with RP were studied. Patient 1 (Pt 1) was a 73-year-old woman. Patient 2 (Pt 2) was a 67-year-old woman. Visual acuity of both patients was light perception. We obtained informed consent from these patients after a full explanation of the purpose of the study and the procedures. The procedures used in this study adhered to the Declaration of Helsinki and were approved by the Ethics Committee of Osaka University Hospital.

\subsection{Retinal prosthesis devices for implantation}

The implanted electronic devices consisted of a secondary coil that receives signals from the external coil and a pulse generator that generates biphasic current pulses to be delivered to the individual electrodes sequentially. The electrode array (size, $5.7 \times 4.6$ $\mathrm{mm}^{2}$; NIDEK, Gamagori, Japan) consisted of 49 electrodes made of 0.5 -mm-diameter platinum (Fig. 1).

\subsection{Surgery}

In both patients, the left eye was selected for the clinical trial. Under local anesthesia with $2 \%$ lidocaine hydrochloride drops, the lateral rectus muscle was dissected at its insertion, and transscleral monopolar stimuli were given to determine the scleral area that has a low threshold to evoke phosphene perception. After the identification and marking of the low-threshold area, general anesthesia was introduced. The skin of the 


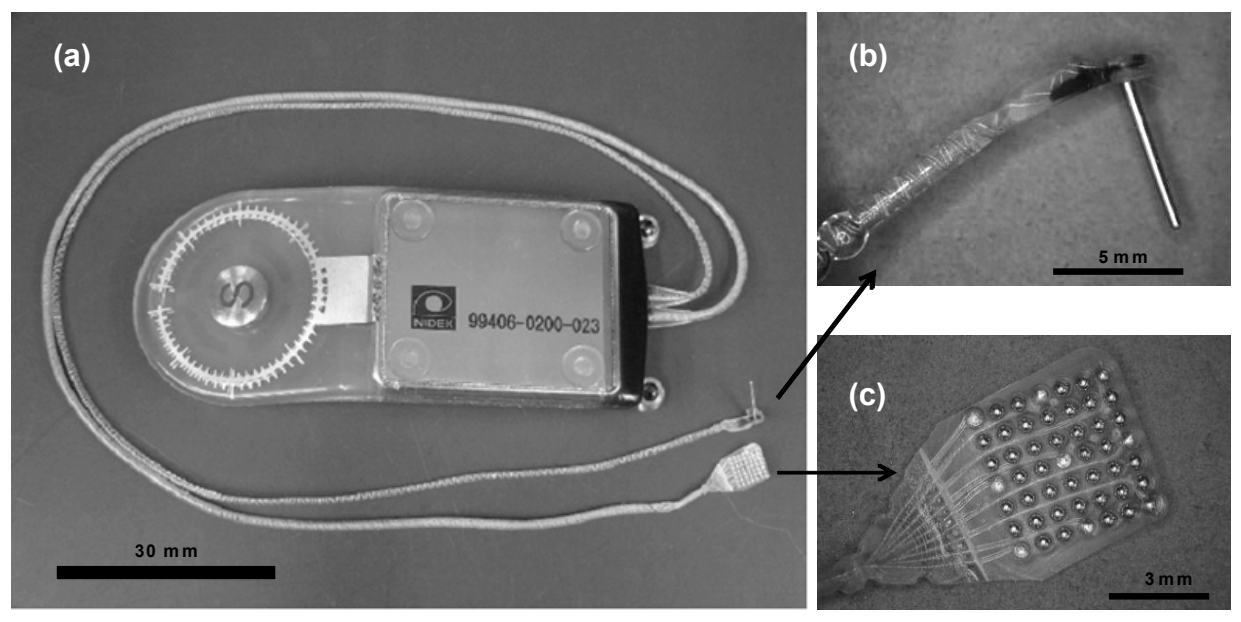

Fig. 1. Photographs of the internal device for STS-retinal prosthesis (a), the electrode array (b), and the return electrode (c).

left temporal area was incised to insert the main part of the internal device [Fig. 1(a)]. The second incision was created at the left zygomatic bone area to fix the lead wire. The electrode array, the return electrode, and the cables were passed under the fascia of the temporal muscle from the first incision to the second incision using a trocar catheter (Medikit, Tokyo, Japan). The bone of the lateral orbital wall was drilled, and the electrode array, the return electrode, and the lead wire were passed into the periocular space using the trocar catheter. The lead wire was fixed by a titanium plate below the second incision. A scleral pocket of $6 \times 5 \mathrm{~mm}^{2}$ was created at the lower temporal scleral area at the lowthreshold area. The electrode array was inserted into the scleral pocket. The return electrode was inserted in the vitreous cavity through the pars plana at the upper nasal area. The lead wires near these electrodes were sutured on the sclera. Five (Pt 1) to seven (Pt 2) weeks after the implantation, all the devices were surgically removed.

\section{$2.4 \quad$ Functional tests}

From one week after the surgery, the wireless system was tested twice a week for 4 weeks. For the functional test of each electrode, 9 out of the 49 electrodes were tested. Cathodic-first biphasic pulses (pulse duration, $0.5 \mathrm{~ms}$; frequency, $20 \mathrm{~Hz}$; interpulse delay, $0.5 \mathrm{~ms}$ ) were delivered through each electrode. We performed the following six visual function tests.

\subsubsection{Test 1: Object detection}

A white box that was $2.6 \times 27 \mathrm{~cm}\left(3.7 \times 34^{\circ}\right.$ visual angle $)$ was set randomly at $15 \mathrm{~cm}\left(21^{\circ}\right)$ to the left or right of the center of the board. The patients were asked where the white box was located. The percentage of correct answers for this task was analyzed statically by the binominal test. We tested whether the percentage was higher than the chance level $(50 \%)$. 


\subsubsection{Test 2: Object discrimination}

Two bars of different widths, $1 \times 30 \mathrm{~cm}\left(1.4 \times 37^{\circ}\right)$ and $3 \times 30 \mathrm{~cm}\left(4.3 \times 3.7^{\circ}\right)$, were presented at the center of the board, and the patients were asked to tell the examiner whether the thicker bar was on the left or right. The percentage of correct answers for this task was analyzed statically by the binominal test. We tested whether the percentage was higher than the chance level (50\%).

\subsubsection{Test 3: Grasping objects}

A white object was randomly set either $15 \mathrm{~cm}\left(21^{\circ}\right)$ to the left or $15 \mathrm{~cm}$ to the right of the center of the board. The patient was asked to grasp the object with her right hand. The percentage of correct answers for this task was analyzed statically by the binominal test. We tested whether the percentage was higher than the chance level (50\%).

\subsubsection{Test 4: Touch panel}

A white rectangular bar of $4.7 \times 20 \mathrm{~cm}\left(6.7 \times 27^{\circ}\right)$ was presented randomly $9.5 \mathrm{~cm}\left(13^{\circ}\right)$ to the left or the right from the center of a touch panel screen (Tyco Electronics, Menlo Park, CA) that was connected to a computer. The patient was asked to touch the white bar with her right index finger. The position touched was recorded and analyzed using the computer. Depending on whether the patient touched the correct position, a different sound was emitted by the computer.

\subsubsection{Test 5: Relationship between pulse frequency and brightness of phosphenes}

We examined the relationship between the brightness of phosphenes and pulse frequency using suprathreshold currents $(0.8 \mathrm{~mA})$ in Pt 2 . The pulse train duration was $1 \mathrm{~s}$. We selected two out of the following frequencies: 10, 20, and $50 \mathrm{~Hz}$. We applied these stimulations to the patient randomly. The patient was asked to compare the brightness of phosphenes evoked by two types of stimulation with different frequencies. Each trial was repeated 4 times.

\section{Results and Discussion}

After the surgery, it was confirmed that the device, cables, and electrodes were implanted and connected, on the basis of skull X-ray projections (Fig. 2). From the fundus picture, fluorescein angiograms, and optical coherence tomography (OCT) images, no significant adverse event was attributed to the implantation surgery in both patients. After the removal surgery, no significant changes were observed in the fundus picture and OCT images. The visual acuity remained at light perception after the removal of the device in both patients. At the removal surgery, the scleral pocket remained stable. Fibrosis or erosion of sclera was not observed around the pocket. The implanted devices and electrode array were kept functional during the 4 weeks of examination and after the removal of the device. Moreover, corrosion was not observed on the surface of electrodes. These results indicated the safety of our STS approach for a retinal prosthesis and also the durability of the implantable device. 


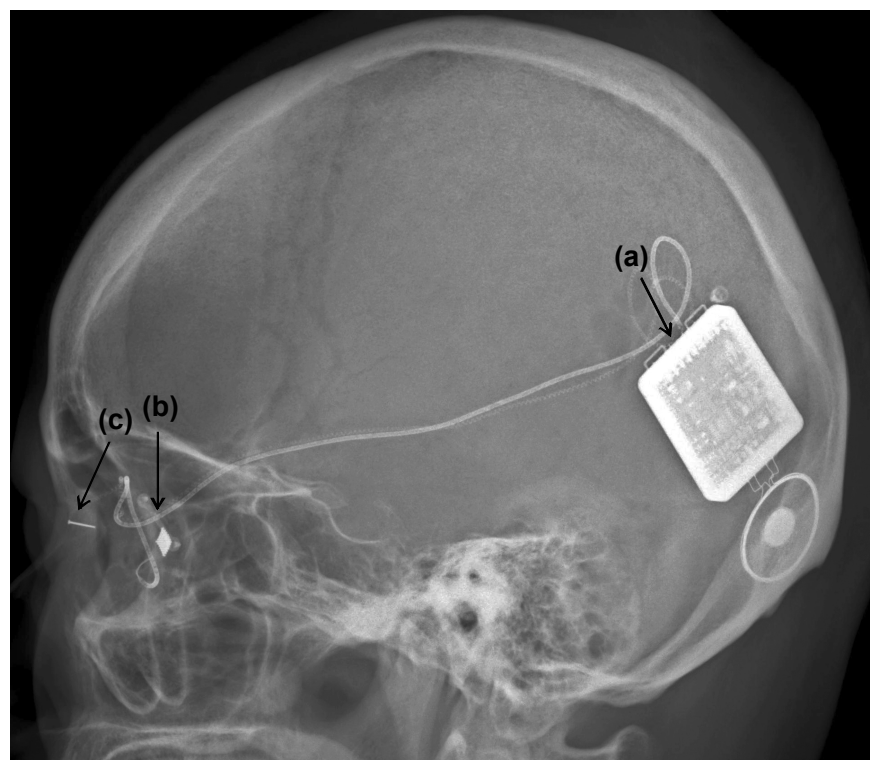

Fig. 2. Lateral view of skull XP of Pt 1. (a) Internal device that has pulse generator circuit and decoder circuit. (b) Electrode array. (c) Return electrode.

Both patients scored better than chance in the object detection and object discrimination tasks with head scanning (Fig. 3). The grasping objects task was carried out by Pt 2 because the elicited phosphene was located close to the subjective center. The score $(90 \%)$ was significantly better than chance. The touch panel task was also applied to only Pt 2. The success rate increased when the testing was repeated (Fig. 4). These functional tests revealed that object discrimination was possible even with a small number of active electrodes.

The subjective phosphenes tended to be brighter at a frequency of $20 \mathrm{~Hz}$ than that at 10 or $50 \mathrm{~Hz}$ (Fig. 5). This test was applied to only Pt 2. This result is consistent with the results of transscleral electrical stimulation. ${ }^{(7)}$ These results suggest that a frequency of around $20 \mathrm{~Hz}$ is the most effective to stimulate the retina of not only a healthy subject but also an RP patient.

\section{Conclusions}

We implanted a retinal prosthesis in two patients with advanced RP. No significant adverse event was observed in either patient after the surgical procedures, confirming the safety of our surgical methods. Functional testing revealed that the detection and discrimination of objects were possible by head scanning with a small number of active 


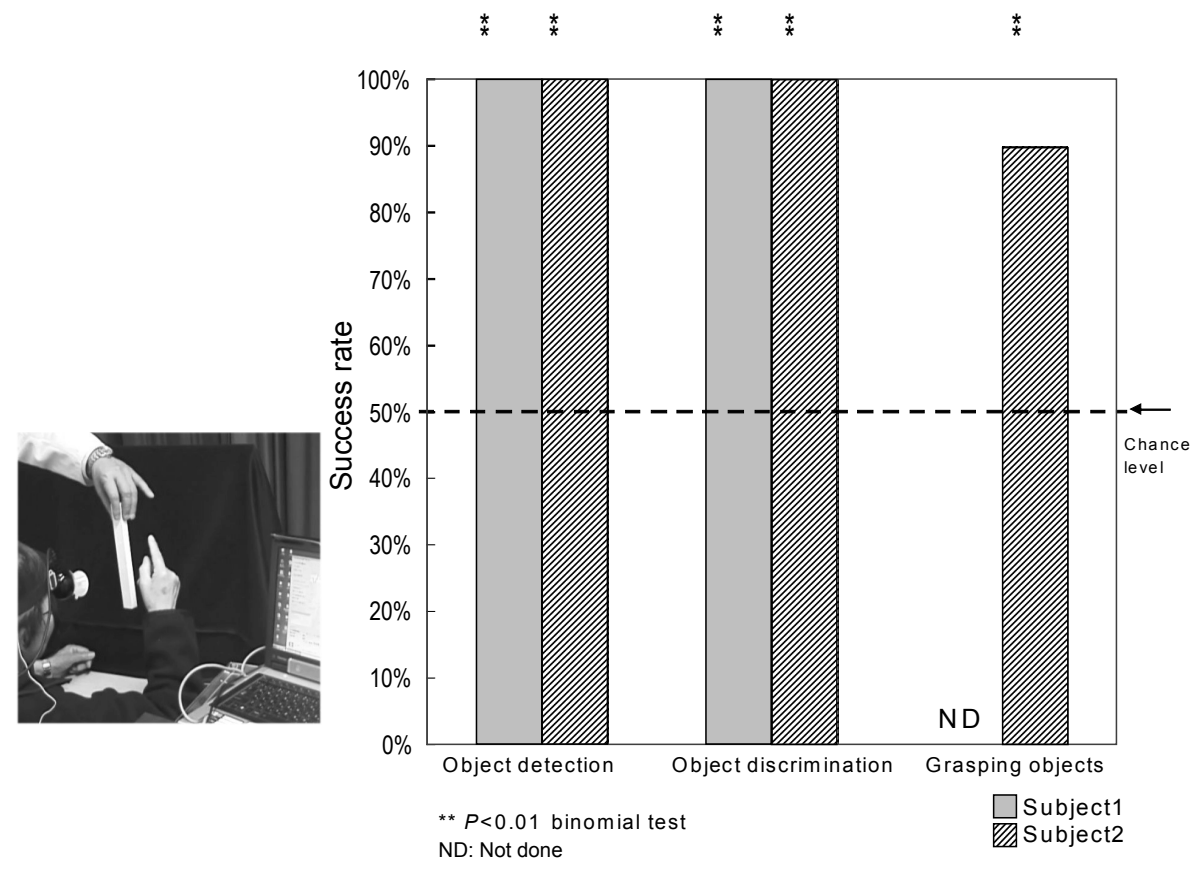

Fig. 3. Success rate of functional test.

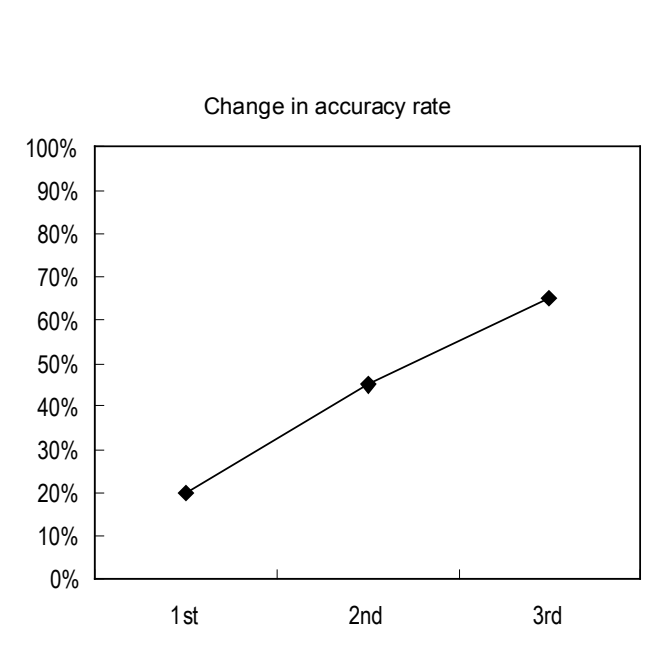

(a)

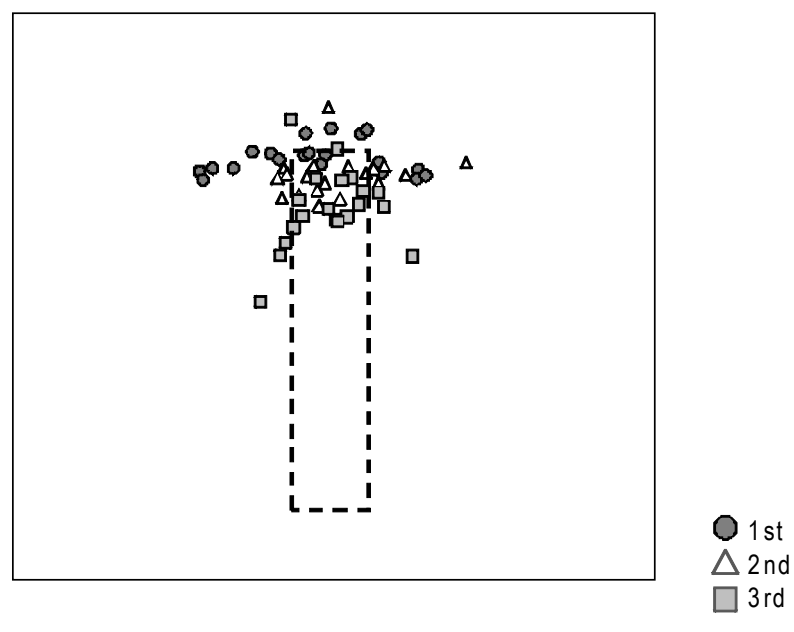

(b)

Fig. 4. Result of the touch panel display task. (a) Changes in success rates after repetition of examination. (b) Touched positions when the white bar was presented on the display. 


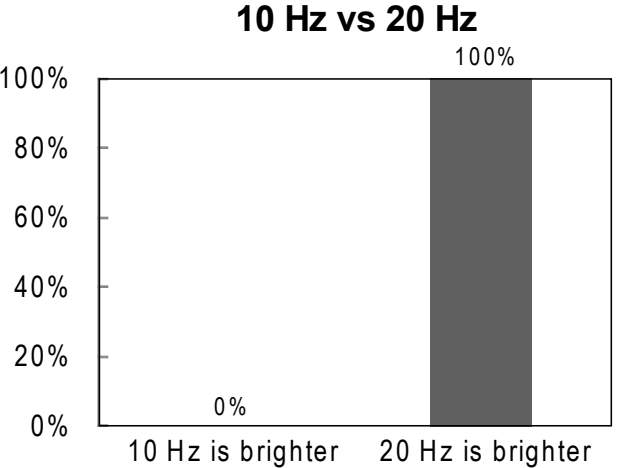

(a)

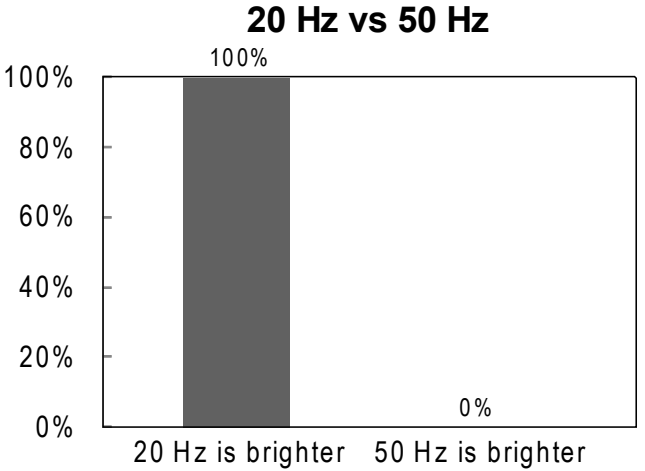

(b)

Fig. 5. Comparison of subjective brightness of phosphene between different pulse frequencies. The patient was asked to state which pulse frequency evoked a brighter phosphene. The trials were repeated 4 times for each parameter set. (a) Comparison between 10 and $20 \mathrm{~Hz}$. (b) Comparison between 20 and $50 \mathrm{~Hz}$.

electrodes. The success rate of identifying a white bar on the touch panel increased after repeated testing. It suggests that a training effect may have occurred during the testing. Chronic implantation of the electrode array-STS system showed that our approach for retinal prosthesis is safe and feasible for artificial vision. Further improvements are necessary to achieve reading ability, and this may require increasing the number of functional electrodes.

\section{References}

1 R. A. Pagon: Surv. Ophthalmol. 33 (1988) 137.

2 E. Zrenner, K. U. Bartz-Schmidt, H. Benav, D. Besch, A. Bruckmann, V. P. Gabel, F. Gekeler, U. Greppmaier, A. Harscher, S. Kibbel, J. Koch, A. Kusnyerik, T. Peters, K. Stingl, H. Sachs, A. Stett, P. Szurman, B. Wilhelm and R. Wilke: Proc. Biol. Sci. 278 (2011) 1489.

3 M. S. Humayun, J. D. Weiland, G. Y. Fujii, R. Greenberg, R. Williamson, J. Little, B. Mech, V. Cimmarusti, G. Van Boemel, G. Dagnelie and E. de Juan: Vision Res. 43 (2003) 2573.

4 H. Kanda, T. Morimoto, T. Fujikado, Y. Tano, Y. Fukuda and H. Sawai: Invest. Ophthalmol. Vis. Sci. 45 (2004) 560.

5 K. Nakauchi, T. Fujikado, H. Kanda, T. Morimoto, J. S. Choi, Y. Ikuno, H. Sakaguchi, M. Kamei, M. Ohji, T. Yagi, S. Nishimura, H. Sawai, Y. Fukuda and Y. Tano: Graefes Arch. Clin. Exp. Ophthalmol. 243 (2005) 169.

6 H. Sakaguchi, T. Fujikado, X. Fang, H. Kanda, M. Osanai, K. Nakauchi, Y. Ikuno, M. Kamei, T. Yagi, S. Nishimura, M. Ohji, T. Yagi and Y. Tano: Jpn. J. Ophthalmol. 48 (2004) 256.

7 T. Fujikado, T. Morimoto, H. Kanda, S. Kusaka, K. Nakauchi, M. Ozawa, K. Matsushita, H. Sakaguchi, Y. Ikuno, M. Kamei and Y. Tano: Graefe's Arch. Clin. Exp. Ophthalmol. 245 (2007) 1411. 